\title{
Kasuistiken
}

Urologe $2021 \cdot 60: 67-70$

https://doi.org/10.1007/s00120-020-01326-2

Online publiziert: 16 . September 2020

(c) Der/die Autor(en) 2020

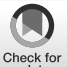

Muhammad Abdeen ${ }^{1}$ Martin Janssen ${ }^{3}$ Zaid Al-Kailani' $\cdot$ Matthias Saar' ${ }^{1}$ Stefan Siemer' ${ }^{1}$ Michael Stöckle ${ }^{1}$ - Gunter Aßmann ${ }^{2}$. Johannes Linxweiler ${ }^{1}$ ${ }^{1}$ Klinik für Urologie und Kinderurologie, Universität des Saarlandes, Homburg/Saar, Deutschland ${ }^{2}$ Klinik für Hämatoonkologie (innere Medizin 1), Universität des Saarlandes, Homburg/Saar, Deutschland ${ }^{3}$ Klinik für Urologie und Kinderurologie, Universitätsklinikum Münster, Münster, Deutschland

\section{Wenn ein urologischer Notfall auf eine internistische Krise hinweist}

\section{Priapismus als klinische Erstmanifestation einer Leukämie}

\section{Falldarstellung}

\section{Anamnese}

Ein junger Patient stellt sich in den frühen Morgenstunden notfallmäßig mit einer seit ca. 24 Stunden (h) bestehenden schmerzhaften Dauererektion ambulant vor. Er berichtete von einem ähnlichen Ereignis, bereits 2 Wochen zuvor. Damals habe sich die Erektion nach einer kalten Dusche wieder spontan zurückgebildet. Es bestehen keine Vorerkrankungen oder Voroperationen, insbesondere auch nicht im kleinen Becken. Regelmäßige Medikamenteneinnahme oder Drogenkonsum werden ebenso verneint, wie Traumata oder Geschlechtsverkehr im Vorfeld der Symptomatik. Die weitere Anamnese ergab eine deutliche B-Symptomatik mit einem Gewichtsverlust von ca. $8 \mathrm{~kg}$ innerhalb der letzten 3 Monate, sowie verstärktes nächtliches Schwitzen.

\section{Klinischer Befund}

Der 32-jährige Patient ist in gutem Allgemein- und schlankem Ernährungszustand: blasses Hautkolorit, Körpertemperatur $37,2^{\circ} \mathrm{C}$, schmerzhafter, vollständig erigierter Penis bei ansonsten unauffälligem äußerem Genitale. Es erfolgt die problemlose beidseitige Punktion der Corpora cavernosa. Der Versuch aus dem Aspirat eine Blutgasanalyse durchzuführen schlägt jedoch wiederholt aus zunächst unbekannten Gründen fehl. Anschließend werden aus den Schwell- körpern ca. $250 \mathrm{ml}$ Blut aspiriert. Nun fällt während der Aspiration auf, dass sich in den gefüllten Spritzen nach kurzer Zeit eine dicklich weiße Schicht von den übrigen Blutbestandteilen separiert (- Abb. 1). Die Laboruntersuchungen des peripher-venös entnommenen Blutes ergeben die in $\bullet$ Tab. 1, 2 und 3 aufgeführten Resultate.

\section{Diagnose}

Low-flow-Priapismus infolge eines Hyperviskositätssyndroms bei hochgradigem Verdacht auf das Vorliegen einer Leukämie.

\section{Therapie und Verlauf}

In den Schwellkörpern werden jeweils $10 \mathrm{mg}$ Etilefrin mit $10 \mathrm{ml} \mathrm{0,9 \%} \mathrm{NaCl}$ verdünnt sowie 1000 IE Heparin injiziert. Hierauf sowie unter manueller Kompression kommt es zu einer kompletten De- tumeszenz. Es wird abschließend für $3 \mathrm{~h}$ ein Druckverband angelegt.

Zur weiteren Diagnostik und Therapie wurde der Patient in die Klinik für Hämatoonkologie aufgenommen. Noch am selben Tag erfolgte eine Beckenkammbiopsie, deren Aufarbeitung letztlich die Diagnose einer chronisch-myeloischen Leukämie $(\mathrm{CML})$ in der chronischen Phase erbrachte. Die molekularzytogenetische Analyse aus den peripheren Blut- und Knochenmarkproben zeigte eine für die CML typische reziproke Translokation zwischen dem langen Arm von Chromosom 9 und dem langen Arm von Chromosom 22 (t[9;22], „Philadelphia-Chromosom"), welche zu einem BCR-ABL1Rearrangement führt. Zusätzlich ließ sich als sog. Major-route-Zusatzaberration eine Trisomie 8 nachweisen, welche jedoch nicht mit einer schlechteren Prognose assoziiert ist [3]. Die abdominelle sonographische Untersuchung zeigt eine Splenomegalie von $22 \times 7 \mathrm{~cm}$, eine signifikante

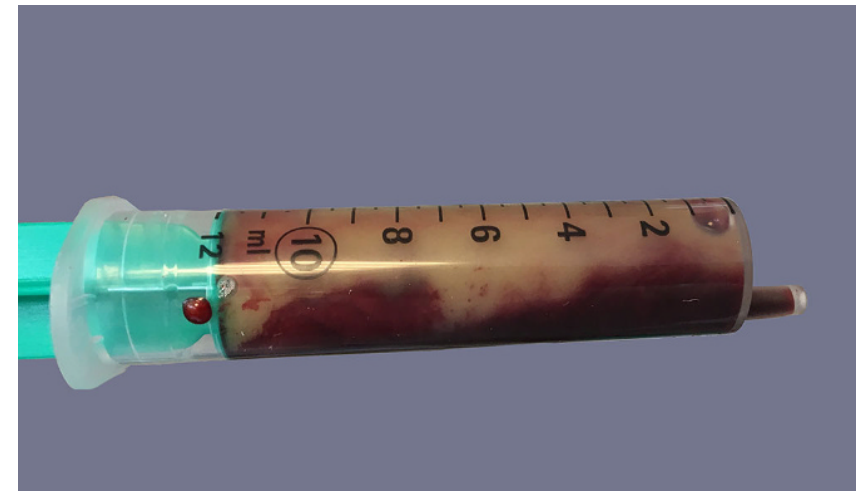

Abb. $1 \varangle$ Aus den Corpora cavernosa aspiriertes Blut 
Hepatomegalie liegt nicht vor. Der EUTOS-Score wurde mit 70 (niedriges Risiko) bestimmt.

Nach der initialen Gabe des Zytostatikums Hydroxycarbamid wurde eine Therapie mit dem Tyrosinkinaseinhibitor Imatinib eingeleitet. Hierunter kam es jedoch nur zu einem unzureichenden Ansprechen, weswegen innerhalb von 4 Wochen auf den Zweitgenerationstyrosinkinaseinhibitor Dasatinib gewechselt wurde. Dies führte $\mathrm{zu}$ einer Normalisierung des Blutbildes innerhalb von 2 Wochen. Aufgrund der nicht abgeschlossenen Familienplanung wurde vor der Einleitung der zytostatischen Therapie dem Patienten eine Kryokonservierung des eigenen Spermas angeboten. Allerdings lehnte der Patient diese ab. Beim letzten Follow-up des Patienten 22 Monate nach Erstdiagnose ist dieser komplett beschwerdefrei und berichtete von einer normalen Erektionsfähigkeit ohne Rezidiv des Priapismus.

\section{Diskussion}

Als Priapismus wird eine persistierende schmerzhafte Erektion $>4 \mathrm{~h}$ bezeichnet, die mit der Gefahr ischämisch bedingter Schädigungen verbunden ist und einen urologischen Notfall darstellt. Dieser kann nach Pathogenese und Klinik in einen ischämischem (IP, Low-flow-Priapismus), einen nicht-ischämischem (NP, High-flow-Priapismus) und einen stotternden Priapismus (SP) unterteilt werden [5]. Neben dem idiopathischen Priapismus sowie den „klassischen“ Ursachen (Traumata, Sichelzellanämie, Einnahme von PDE-V-Hemmern, SKATTherapie und Psychopharmaka) kann der Priapismus auch Zeichen einer lebensbedrohlichen Systemerkrankung sein. Der maligne Priapismus (MP) ist eine Sonderform des IP, der im Rahmen verschiedener neoplastischer Erkrankungen auftreten kann, so bei diversen soliden aber auch hämatoonkologischen Tumorerkrankungen [2].

Die CML ist eine myeloproliferative Erkrankung, die durch das Vorhandensein der Translokation t(9;22) und der damit einhergehenden Entstehung des $B C R$ - $A B L$-Fusionsgens gekennzeichnet ist (das hierdurch verkürzte Chro- mosom 22 wird auch als „PhiladelphiaChromosom“ bezeichnet). In der Regel erfolgt die Erstvorstellung von CML-Patienten mit laborchemischer Leukozytose, Hepatosplenomegalie sowie unspezifischen Symptomen wie Fieber, Müdigkeit und Gewichtsverlust. In sehr seltenen Fällen entwickelt sich in Folge der Hyperviskosität des Blutes bei der CML ein maligner Priapismus (<3\%) [1]. Noch seltener ist der MP klinische Erstmanifestation der CML. Grundsätzlich beinhaltet das Therapiekonzept bei dieser seltenen klinischen Konstellation die notfallmäßige Behandlung des MP und anschließend die umgehende Initiierung einer Therapie der Grunderkrankung. Nach Anamneseerhebung und klinischer Untersuchung gelten die Punktion der Corpora cavernosa mit Blutgasanalyse des Aspirats und eine Labordiagnostik evtl. inklusive Tumormarkerbestimmung als wichtige initiale diagnostische und therapeutische Schritte beim MP. Führt die Aspiration des Blutes aus den Corpora caveronosa alleine nicht zur Detumeszenz ist die Injektion von vasoaktiven Substanzen (z. B. Phenylephrin) und Heparin angezeigt. Die chirurgische Intervention im Sinne der Anlage einer artifiziellen Fistel zwischen Corpora cavernosa und Glans penis (z. B. Winter-Shunt) ist erst nach Ausschöpfung dieser konservativen Maßnahmen indiziert [7].

Pathophysiologisch entsteht aufgrund der Leukozytose bei der CML eine Hyperviskosität des Blutes, welche zu einer Aggregation von Leukämiezellen im Schwellkörper und konsekutiv zu einem Versagen der physiologischen Detumeszenz führt. Außer der CML wurden die Sichelzellanämie, die chronischelymphatische Leukämie (CLL) und die akute lymphatische Leukämie (ALL) als andere hämatologische Ursachen des Priapismus beschrieben [6].

Obwohl beim IP von $24 \mathrm{~h}$ Dauer in den europäischen Leitlinien ein hohes Risiko einer erektilen Dysfunktion beschrieben wird [5], wurde diese in unserem Fall nicht beobachtet.

Diese Arbeit ist nicht die erste, die vom Auftreten eines Priapismus bei CML berichtet. Unser Fallbericht gehört allerdings zu den wenigen, die vom Priapismus als klinischer Erstmanifestation ei-
Urologe 2021 · 60:67-70

https://doi.org/10.1007/s00120-020-01326-2

(c) Der/die Autor(en) 2020

M. Abdeen - M. Janssen · Z. Al-Kailani ·

M. Saar · S. Siemer - M. Stöckle •

G. Aßmann · J. Linxweiler

Wenn ein urologischer Notfall auf eine internistische Krise hinweist. Priapismus als klinische Erstmanifestation einer Leukämie

\section{Zusammenfassung}

Der Priapismus als klinische Manifestation einer hämatologischen Erkrankung ist selten. In diesem Fall liegt ein sowohl urologischer als auch internistischer Notfall vor, der einer umgehenden Therapie bedarf. Dieser Artikel beschreibt den klinischen Fall eines Priapismus als Erstmanifestation einer bis dahin nicht diagnostizierten chronischmyeloischen Leukämie (CML) und erläutert die Resultate einer Literaturrecherche zu dieser Thematik.

\section{Schlüsselwörter}

Philadelphia-Chromosom · Maligner

Priapismus · Hyperviskositätssyndrom .

Chronisch-myeloische Leukämie · Fallbericht

When a urological emergency indicates an internal medical crisis. Priapism as the first clinical manifestation of leukemia

\section{Abstract}

Priapism as a sign of a severe hematological disease is a rare event, which has to be considered as both a urological and a hematological emergency that requires immediate treatment. This article describes a clinical case of priapism as the first clinical manifestation of a hitherto undiagnosed chronic myeloid leukemia (CML) and discusses the results of a literature review on this topic.

Keywords

Philadelphia chromosome $\cdot$ Malignant priapism $\cdot$ Hyperviscosity syndrome $\cdot$ Chronic myeloid leukemia. Case report 
Tab. 1 Kleines Blutbild

\begin{tabular}{lll}
\hline Parameter & Wert & Referenzbereich \\
\hline Leukozyten & $422.500 / \mu \mathrm{l}$ & $3900-10.200 / \mu \mathrm{l}$ \\
\hline Erythrozyten & $2,76 \times 10^{6} / \mu \mathrm{l}$ & $4,5-5,9 \times 10^{6} / \mu \mathrm{l}$ \\
\hline Hämoglobin & $8,8 \mathrm{~g} / \mathrm{dl}$ & $14-18 \mathrm{~g} / \mathrm{dl}$ \\
\hline Hämatokrit & $24 \%$ & $41-53 \%$ \\
\hline Thrombozyten & $173.000 / \mu \mathrm{l}$ & $140.000-400.000 / \mu \mathrm{l}$ \\
\hline Mittleres Zellvolumen der Erythrozyten & $87 \mathrm{fl}$ & $80-99 \mathrm{fl}$ \\
\hline Mittleres Korpuskuläres Hämoglobin & $32 \mathrm{pg}$ & $27-33 \mathrm{pg}$ \\
\hline Mittlere korpusukuläre Hämoglobin-Konzentration & $37 \mathrm{~g} / \mathrm{dl}$ & $31-37 \mathrm{~g} / \mathrm{dl}$ \\
\hline Erythrozytenverteilungsbreite & $18,7 \%$ & $11,5-14,5 \%$ \\
\hline Mittleres Thrombozytenvolumen & $10,5 \mathrm{fl}$ & $7,8-11,0 \mathrm{fl}$ \\
\hline Unreife Thrombozyten & $2,2 \%$ & $1,1-6,1 \%$
\end{tabular}

\section{Tab. 2 Klinische Chemie}

\begin{tabular}{ll|l}
\hline Parameter & Wert & Referenzbereich \\
\hline Natrium & $136 \mathrm{mmol} / \mathrm{l}$ & $135-145 \mathrm{mmol} / \mathrm{l}$ \\
\hline Kalium & $4,5 \mathrm{mmol} / \mathrm{I}$ & $3,5-5-1 \mathrm{mmol} / \mathrm{l}$ \\
\hline Chlorid & $96 \mathrm{mmol} / \mathrm{l}$ & $98-107 \mathrm{mmol} / \mathrm{l}$ \\
\hline Kalzium & $2,3 \mathrm{mmol} / \mathrm{I}$ & $2,2-2,6 \mathrm{mmol} / \mathrm{l}$ \\
\hline Kreatinin & $1,20 \mathrm{mg} / \mathrm{dl}$ & $0,70-1,20 \mathrm{mg} / \mathrm{dl}$ \\
\hline Harnstoff & $32 \mathrm{mg} / \mathrm{dl}$ & $17-48 \mathrm{mg} / \mathrm{dl}$ \\
\hline Harnsäure & $8,0 \mathrm{mg} / \mathrm{dl}$ & $3,4-7,0 \mathrm{mg} / \mathrm{dl}$ \\
\hline Glukose & $105 \mathrm{mg} / \mathrm{dl}$ & $60-100 \mathrm{mg} / \mathrm{dl}$ \\
\hline C-reaktives Protein & $14,8 \mathrm{mg} / \mathrm{l}$ & $<5,0 \mathrm{mg} / \mathrm{l}$ \\
\hline Laktatdehydrogenase & $1.237 \mathrm{U} / \mathrm{l}$ & $0-262 \mathrm{U} / \mathrm{l}$
\end{tabular}

\section{Tab. 3 Differentialblutbild}

\begin{tabular}{|l|l|l|}
\hline Parameter & Wert & Referenzbereich \\
\hline Neutrophile & $80 \%$ & $42-77 \%$ \\
\hline Neutrophile (abs.) & 371,3 & $1500-7700 / \mu \mathrm{l}$ \\
\hline Basophile & $6 \%$ & $0-1 \%$ \\
\hline Esinophile & $4 \%$ & $0-5 \%$ \\
\hline Lymphozyten & $4 \%$ & $25-45 \%$ \\
\hline Monozyten & $6 \%$ & $2-10 \%$ \\
\hline Blasten undifferenziert & $2 \%$ & - \\
\hline Kernschatten & $11,8 \%$ & $0 \%$ \\
\hline
\end{tabular}

ner CML berichten und dies zudem mit eindrücklichem Bildmaterial untermauern können. Weiterhin gehen wir ausführlich auf die für die Praxis relevanten Therapiealgorithmen ein.

Aufgrund der Seltenheit dieses Falles gibt es für den leukämischen Priapismus keine empfohlene Standarttherapie. Prinzipiell soll die Therapie aus einer lokalen Notfallbehandlung des Priapismus dicht gefolgt von der Einleitung einer systemischen Therapie der CML bestehen. Nach den Leitlinien der American Uro- der Hyperviskosität des aspirierten Blutes appliziert.

\section{Fazit für die Praxis}

- Es kann neben den „klassischen“ auch sehr seltene Ursachen eines Priapismus geben, an welche differentialdiagnostisch gedacht werden muss.

- Bei diesen seltenen Fällen kann der Priapismus sogar die klinische Erstmanifestation einer lebensbedrohlichen Erkrankung sein.

\section{Korrespondenzadresse}

Dr. med.
Muhammad Abdeen
Klinik für Urologie und
Kinderurologie, Universität
des Saarlandes
Kirrberger Straße, Gebäude 6,
66424 Homburg/Saar,
Deutschland
muhammad.abdeen@uks.eu

Funding. Open Access funding provided by Projekt DEAL.

\section{Einhaltung ethischer Richtlinien}

Interessenkonflikt. M. Abdeen, M. Janssen, Z. Al Kailani, M. Saar, S. Siemer, M. Stöckle, G. Aßmann und J. Linxweiler geben an, dass kein Interessenkonflikt besteht.

Dieser Beitrag beinhaltet keine Studien an Menschen oder Tieren. Für die aufgeführten Studien gelten die jeweils dort angegebenen ethischen Richtlinien. Der Patient wurde über die anonymisierte Publikation seines Falles inklusive Bildmaterial informiert und hat dieser schriftlich zugestimmt.

Open Access. Dieser Artikel wird unter der Creative Commons Namensnennung 4.0 International Lizenz veröffentlicht, welche die Nutzung, Vervielfältigung, Bearbeitung, Verbreitung und Wiedergabe in jeglichem Medium und Format erlaubt, sofern Sie den/die ursprünglichen Autor(en) und die Quelle ordnungsgemäß nennen, einen Link zur Creative Commons Lizenz beifügen und angeben, ob Änderungen vorgenommen wurden

Die in diesem Artikel enthaltenen Bilder und sonstiges Drittmaterial unterliegen ebenfalls der genannten Creative Commons Lizenz, sofern sich aus der Abbildungslegende nichts anderes ergibt. Sofern das betreffende Material nicht unter der genannten Creative Commons Lizenz steht und die betreffende Handlung nicht nach gesetzlichen Vorschriften erlaubt ist, ist für die oben aufgeführten Weiterverwendungen des Materials die Einwilligung des jeweiligen Rechteinhabers einzuholen. 


\section{Kasuistiken}

Weitere Details zur Lizenz entnehmen Sie bitte der Lizenzinformation auf http://creativecommons.org/ licenses/by/4.0/deed.de.

\section{Literatur}

1. Becerra-Pedraza LC (2018) Priapism as the initial sign in hematologic disease: case report and literature review. Int JSurg Case Rep 43:13-17

2. De Luca F (2016) Malignant priapism due to penile metastases: case series and literature review. Arch Ital Urol Androl 88(2):150-152

3. Fabarius A (2011) Impact of additional cytogenetic aberrations at diagnosis on prognosis of CML: long term observation of 1151 patients from the randomized CML Study IV. Blood 118(26):6760-6768

4. Montague DK (2003) American Urological Association guideline on the management of priapism. JUrol 170:1318-1324

5. Salonia A (2014) European Association of Urology guidelines on priapism. Eur Urol 65(2):480-489

(seit 2014 erfolgte keine Aktualisierung der Leitlinie)

6. Schreibman SM (1974) Management of priapism in patients with chronic granulocytic leukemia.JUrol 111:786-788

7. Winter CC (1976) Cure of idiopathic priapism: new procedure for creating fistula between glans penis and corpora cavernosa. Urology 8(4):389-391

\section{Hier steht eine Anzeige.}

\section{Springer}

